\title{
A Traveling Salesman Problem for a Class of
}

\section{Carrier-Vehicle Systems}

\author{
Emanuele Garone ${ }^{1}$ \\ Université Libre de Bruxelles, B-1050 Brussels, Belgium \\ Roberto Naldi ${ }^{2}$ \\ University of Bologna, Bologna (BO), 40136, Italy \\ Alessandro Casavola ${ }^{3}$ \\ University of Calabria, Arcavacata di Rende (CS), 87036, Italy
}

This work addresses a Traveling Salesman Problem for a class of systems composed of two cooperating vehicles with different capabilities. The first one, the carrier, is typically slow but has a virtually infinite operating range. Its primary role is to deploy and recover a second vehicle, typically an aircraft, which is faster but has a reduced operating range. The synergistic use of the above carrier-vehicle system is exploited in addressing a scenario in which the goal is to make the faster vehicle visit in the shortest time a given collection of targets. After a careful analysis of the problem, a sub-optimal heuristic is presented and its properties are discussed.

\section{Introduction}

The complexity of many scenarios envisioned for future autonomous systems, ranging from planetary exploration to rescue missions, requires a broad range of capabilities for individual unitsoften including air, ground or sea mobility and sophisticated multi-modal sensor suites and actuation devices - which cannot be implemented with a single platform class. Rather, it may be necessary

\footnotetext{
1 Assistant Professor, Departments of Control and System Theory. egarone@ulb.ac.be

2 Associate Researcher, Department of Electronics, Computer Sciences and Systems. roberto.naldi@unibo.it.

${ }^{3}$ Full Professor, Department of Electronics, Computer Sciences and Systems. casavola@deis.unical.it
} 
to coordinate several specialized units to attain complex objectives in a reliable, timely, and efficient way [1]. While considerable progress has been made on cooperative control of networks of homogeneous vehicles (see for example [2-5]), heterogeneous networks are still relatively poorly understood. In such a direction, recent efforts (see e.g. [6] and [7]) have been undertaken for spreading the adoption of unmanned systems in real-world operational scenarios. In particular see [8] where the employment of cooperating mobile robots, often denoted as multiple mobile robot systems, is a clear example of the capabilities achievable by combining the characteristics of heterogeneous vehicles with complementary features. In order to optimally exploit the different capabilities of each individual unit in obtaining the desired final behavior the team is required to be suitably coordinated via advanced planning and control algorithms.

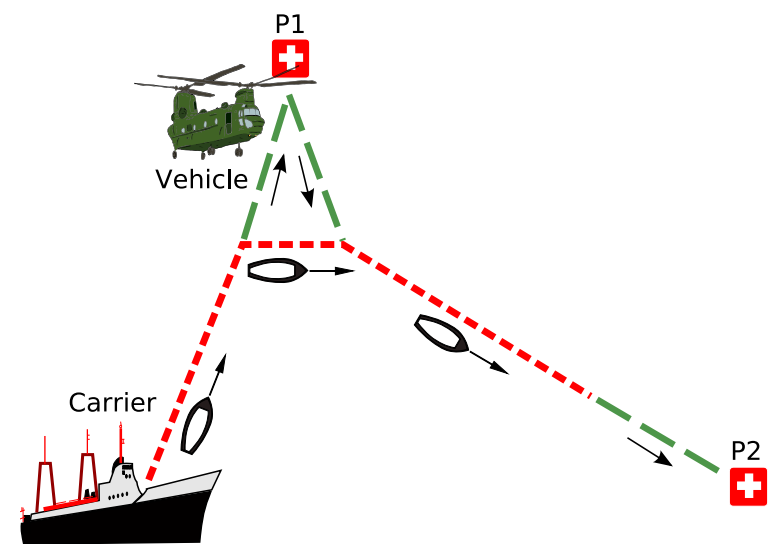

Fig. 1 The carrier-vehicle system on a rescue scenario.

This note concentrates on systems of heterogeneous vehicles, arising from the combination of (i) a slow autonomous carrier vehicle (typically a ship) with a long operating endurance and (ii) a faster vehicle (typically an aircraft) with a limited operative endurance. The carrier is able to transport the faster vehicle, as well as to deploy, recover, and service it. Even though this two-vehicle system is very simple it has relevant applications and many path planning and coordination problems of interest - similar to those introduced in [9-11] for other systems - may be defined for it. In the preliminary work [12] the determination of the optimal trajectories connecting up to 2 given points has been detailed. This note extends previous results to the case in which $n$ points have to be visited and the visiting sequence is not a priori given. 


\section{The Carrier-Vehicle System}

The system we are going to deal with is composed of two different vehicles: a carrier, whose variables and functions will be denoted by the subscript $\cdot_{c}$, and a vehicle, denoted by the subscript ${ }_{v}$. In the following we will refer to the combined system as the carrier-vehicle system. The vehicles will be considered as material points belonging to the Euclidean space $\mathbb{R}^{2}$ and their position will be denoted respectively as $p_{c}(t)=\left[x_{c}(t) y_{c}(t)\right]^{T}$ and $p_{v}(t)=\left[x_{v}(t) y_{v}(t)\right]^{T}$. It is assumed that the carrier has a single integrator behavior and thus it is able to follow any continuous path with a speed that is limited to be lower or equal than a certain maximal velocity $V_{c}>0$, i.e. $\left\|\dot{p}_{c}(t)\right\|:=$ $\sqrt{\dot{x}_{c}(t)^{2}+\dot{y}_{c}(t)^{2}} \in\left[0, V_{c}\right]$ for all $t \geq 0$. For what regards the carried vehicle we need to distinguish between two different situations:

- when it is not carried, it can follow any continuous path with a speed lower or equal than $V_{v}>V_{c}$, i.e. $\left\|\dot{p}_{v}(t)\right\|:=\sqrt{\dot{x}_{v}(t)^{2}+\dot{y}_{v}(t)^{2}} \in\left[0, V_{v}\right]$

- when it is carried, its position coincides with that of the carrier, viz. $p_{v}(t)=p_{c}(t)$.

In order to model the limited operating range of the faster vehicle, it is assumed that it can leave the carrier position, i.e. $p_{v} \neq p_{c}$, for no more time than $\bar{a}$ units of time. Moreover, any time the vehicle comes back to the carrier, i.e. $p_{v} \equiv p_{c}$, its remaining operating endurance is instantaneously restored to $\bar{a}$.

\section{Ordered visit of $n$ points}

Among the large variety of planning problems that can be formulated considering the above presented carrier-vehicle system, this note will focus on a special scenario inspired in particular by rescue operations, see for instance [7], where the faster vehicle is required to land back to the carrier after having visited each target point (e.g. to transport victims on the main vehicle as soon as possible). Within this framework the first mission planning problems we can define is the following:

Problem 1 - Let an initial point $p_{0}$, a desired terminal point $p_{f}$ and a list of $n$ points $q_{l i s t}=$ $\left[q_{1}, \ldots, q_{n}\right]$ be given with $p_{c}(0)=p_{v}(0)=p_{0}$. Determine the minimum-time trajectory allowing each point to be visited by the carried vehicle in an ordered way by respecting, for each point $q_{i}$, a given sequence of takeoff - visiting the new point- landing prescriptions and ending, for both vehicles, at 
the terminal point $p_{f}$.

By exploiting the fact that, in the absence of constraints, the optimal trajectory between two points is a straight line covered at the maximal speeds for both the carrier and the carried vehicle, the above problem may be formulated as the following optimization problem:

$$
\begin{array}{lr}
\min _{p_{t o, i}, p_{l, i}, t_{p_{l, i}, p_{t o, i+1},}, t_{p_{t o, i}, p_{l, i}}} \sum_{i=0}^{n} t_{p_{l, i}, p_{t o, i+1}}+\sum_{i=1}^{n} t_{p_{t o, i}, p_{l, i}} \\
t_{p_{t o, i}, p_{l, i}} \leq \bar{a}, & i=1, \ldots, n \\
\frac{1}{V_{c}}\left\|p_{l, i}-p_{t o, i}\right\| \leq t_{p_{t o, i}, p_{l, i}}, & i=1, \ldots, n \\
\frac{1}{V_{v}}\left(\left\|q_{i}-p_{t o, i}\right\|+\left\|p_{l, i}-q_{i}\right\|\right) \leq t_{p_{t o, i}, p_{l, i}}, & i=1, \ldots, n \\
\frac{1}{V_{c}}\left\|p_{l, i}-p_{t o, i+1}\right\| \leq t_{p_{l, i}, p_{t o, i+1}}, & i=0, \ldots, n
\end{array}
$$

where the unknowns to be determined are the collection of points $p_{t o, i}, p_{l, i} \in \mathbb{R}^{2}, i \in\{1, \ldots, n\}$, representing the take-off and the landing points that allow the vehicle to optimally visit the $i$-th target, and the time intervals $t_{p_{l, i}, p_{t o, i+1}} \in \mathbb{R}, t_{p_{t o, i}, p_{l, i}}, i \in\{1, \ldots, n\}$ representing, respectively, the time that the carrier will employ to reach the $(i+1)$-th take-off point from the $i$-th landing point and the time that the vehicle will spend between the take-off and the subsequent landing instants needed to visit the $i$-th point.

For simplicity, $p_{l, 0}=p_{0}$ and $p_{t o, n+1}=p_{f}$ will be assumed. Note that, since the objective function is linear and the constraints are convex, (1) is a convex optimization problem and then the optimum may be efficiently computed through numerical solvers.

Although an exact analytic solution is unknown (beside a few special cases like those discussed in [12]), it is possible to analytically determine upper-bounds and lower bounds to the optimal solution of Problem 1. Namely, it is possible to prove that a lower bound to the optimal cost of Problem 1 is always given by

$$
t_{L}(\ell, n)=\max \left\{\left(\ell / V_{c}-n V_{v} \bar{a} / V_{c}+n \bar{a}\right), \ell / V_{v}\right\}
$$

where $\ell$ is the length of the shortest path that a single holonomic vehicle would undertake to complete the visit of all points of interest, i.e. $\ell=\sum_{i=1}^{n+1} d_{i-1, i}$, where $d_{0,1}:=\left\|p_{0}-q_{1}\right\|, d_{i-1, i}:=\| q_{i-1}-$ $q_{i}\left\|, i \in\{2,3, \ldots n\}, d_{n, n+1}:=\right\| q_{n}-p_{f} \|$. 
In order to derive an upper bound it is enough to find analytically a feasible, not necessarily optimal, solution to Problem 1. To this end, denote $d_{m i n}:=\min _{i=1, \ldots, n} d_{i-1, i}$ and let $\bar{a}^{\prime}=\min \left\{d_{\min } / V_{v}, \bar{a}\right\}$. The following upper bound

$$
t_{U}\left(\ell, n, \theta_{\text {list }}\right)=t_{L}(\ell, n)+\sum_{i=1}^{n} \frac{\Delta\left(\theta_{i}, \bar{a}, \bar{a}^{\prime}\right)}{V_{c}}
$$

may be obtained with

$$
\Delta\left(\theta, \bar{a}, \bar{a}^{\prime}\right):=\left\{\begin{array}{c}
\left(\bar{a}-\bar{a}^{\prime}\right) V_{v} \quad \text { if } \theta \leq 2 \arcsin \left(\frac{V_{c}}{V_{v}}\right) \\
\bar{a} V_{v}-\bar{a}^{\prime} V_{c} / \sin (\theta / 2) \text { else }
\end{array}\right.
$$

where $\theta_{\text {list }}:=\left[\theta_{1}, \theta_{2}, \ldots \theta_{n}\right], \theta_{i} \in[0, \pi], i=1, \ldots, n-1$, denotes the list of the $n$ (smallest) angles formed by the segments that connect two consecutive points to be visited (for a graphical intuition see also Figure 2). Please note that if $\bar{a}^{\prime}=\bar{a}$ and $\theta_{i} \leq 2 \arcsin \left(V_{c} / V_{v}\right)$ for all $\theta_{i} \in \theta_{\text {list }}$, the proposed upper bound precisely matches the lower bound (2) and indeed it is the optimal cost.

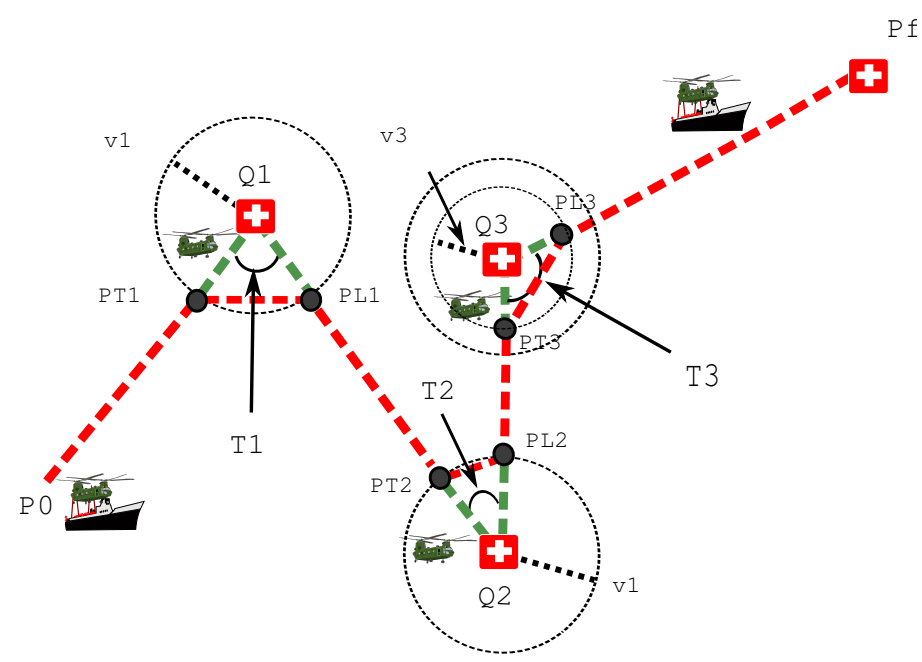

Fig. 2 The geometric interpretation behind the upper bound $t_{U}\left(\ell, n, \theta_{\text {list }}\right)$.

\section{The Traveling Salesman Problem}

In this note we are interested in dealing with the following Carrier-Vehicle Traveling Salesman Problem (CV-TSP):

Problem 2 (CV-TSP) - Let $p_{0}$ be the same initial position for both vehicles, viz. 
$p_{c}(0)=p_{v}(0)=p_{0}$, and assume an unordered set of $n$ points $q_{\text {set }}=\left\{q_{1}, \ldots, q_{n}\right\}$ to be visited be given. Determine the minimum-time trajectory allowing each point to be visited by the carried vehicle by respecting, for each point $q_{i}$ to be visited, a given sequence of takeoff - visiting the new point- landing prescriptions and ending, for both vehicles, to the initial point $p_{0}$.

As well known, Traveling Salesman Problems are typical NP-Hard optimization problems. For such a reason, in most practical cases, heuristics have to be used to solve them. In this note, in order to deal with the particular TSP problem at hands, we propose a heuristic algorithm based on the Euclidean TSP (E-TSP). E-TSP is a particular case of the general TSP problem in which, given $n$ points in the space, the goal is to determine the optimal sequence that minimizes the sum of the Euclidean distances between consecutive points. One of the main feature of this class of TSP problems is that, although still NP-Hard, they admit polynomial-in-time optimization schemes (see [13]). This means that, for any scalar $e>0$, it is possible to find in a polynomial time a tour whose length is at most $(1+1 / e)$ times larger than the optimal length. Then, in practice, for any instance of E-TSP we can obtain an almost-optimal solution in a reasonable time. The CV-TSP heuristic here proposed consists of the following two steps:

1. determine the visiting order of the almost-optimal E-TSP tour for the set of given points $\left\{p_{0}\right\} \cup q_{\text {set }} ;$

2. Use the above visiting order to solve the resulting visit of $n$ points (Problem 1 ) via the convex optimization formulation (1).

The idea behind this approach is that the completion time of the CV-TSP is related to the sum of the distances between points and thus the minimization of E-TSP leads usually to achieve a reasonably good CV-TSP solution. In particular, it is possible to prove that:

Lemma 1 - Let the initial point $p_{0}$ and the set of $n$ points $q_{\text {set }}$ to be visited be given. Let $\ell_{\text {ETSP }}$ denote the length of the $(1+1 / e)$-approximated optimal E-TSP tour, with $e \geq 0$, and $\theta_{\text {list }}^{E T S P}:=\left[\theta_{1}, \theta_{2}, \ldots \theta_{n}\right], \theta_{i} \in[0, \pi], i=1, \ldots, n-1$ the list of the $n$ (smallest) angles formed by the 
segments connecting two consecutive points in the order given by the approximated E-TSP solution.

Then, the completion time $t_{C V-T S P}^{\text {heu }}$ obtained with the proposed CV-TSP heuristic has a cost which is at most $\varepsilon$ times greater than the optimal one, with $\varepsilon$ given by

$$
\varepsilon:=\frac{t_{U}\left(\ell_{E T S P}, n, \theta_{\text {list }}^{\text {ETSP }}\right)}{t_{L}\left(\frac{\ell_{E T S P}}{1+1 / e}, n\right)}
$$

where $t_{L}(\cdot)$ and $t_{U}(\cdot)$ are defined in (2) and (3).

Proof - By recalling the definition of the lower bound (2), for any given number of points $n$ the implication $\ell_{1} \leq \ell_{2} \Rightarrow t_{L}\left(\ell_{1}, n\right) \leq t_{L}\left(\ell_{2}, n\right)$ holds true. Then, let $\ell$ denote the length of a generic Hamiltonian cycle for the set of points $p_{0} \cup q_{\text {set }}$ and $\ell_{E T S P}^{\text {opt }}$ the length of the optimal E-TSP solution. It follows that $\ell_{E T S P}^{o p t} \leq \ell$. As a consequence, $t_{L}\left(\ell_{E T S P}^{o p t}, n\right) \leq t_{L}(\ell, n)$ which implies that $t_{L}\left(\ell_{E T S P}^{o p t}, n\right)$ is a lower bound to the optimal solution of CV-TSP. Moreover, by the property of the $(1+1 / e)$-approximated optimal E-TPS solution, it follows that $\ell_{E T S P}^{o p t} \geq \ell_{E T S P} /(1+1 / e)$, being $t_{L}\left(\frac{\ell_{E T S P}}{1+1 / e}, n\right)$ a lower bound to the optimal solution of CV-TSP as well. The proof ends by noticing that $t_{U}\left(\ell_{E T S P}, n, \theta_{\text {list }}^{E T S P}\right)$ is an upper bound to the proposed heuristic solution.

Please note that, for the properties of the upper bound to Problem 1, in the particular case the points to be visited are sufficiently far from one another (i.e. $d_{\min } / V_{v}>\bar{a}$ ) and the angles $\theta_{i}$ formed by the segments connecting the points in the order given by the ETSP algorithm satisfy $\theta_{i} \leq 2 \arcsin \left(V_{c} / V_{v}\right)$, than $\varepsilon=t_{L}\left(\ell_{E T S P}, n\right) / t_{L}\left(\frac{\ell_{E T S P}}{1+1 / e}, n\right)$ results and the optimal sequence of points for ETSP is also optimal for CV-TSP.

Numerical Results - Numerical simulations have been undertaken to compare the optimal solution of CV-TSP with the one obtained by the proposed heuristic in a realistic scenario in which a combined USV/UAV (Unmanned Surface Vehicle / Unmanned Aerial Vehicle) system has to monitor some randomly generated points of interest in a certain portion of sea. The USV, which represents the carrier, is assumed to have a maximum velocity $V_{c}=10 \mathrm{mph}$ while the aircraft has a maximum flight speed $V_{v}=50 \mathrm{mph}$ and a flight endurance $\bar{a}=0.4$ hours.

The targets to be visited are limited to 5 randomly generated points which still make the computation of the exact optimal solution in a reasonable time possible. As highlighted in Lemma 1, the distances among targets play an important role in the performance of the proposed CV-TSP 


\begin{tabular}{|c|c|c|c|c|}
\hline & Cases \# & Opt. Sol. & Avg Degradation & Max Degradation \\
\hline LD & 500 & $88.2 \%$ & $0.028 \%$ & $1.48 \%$ \\
ND & 1000 & $73.1 \%$ & $0.104 \%$ & $7.5 \%$ \\
SD & 500 & $52 \%$ & $0.526 \%$ & $25.1 \%$ \\
\hline
\end{tabular}

Table 1 Comparison between the optimal solution of CV-TSP and the proposed heuristic.

heuristic. In this respect, the simulations consider three different scenarios which differ by the size of the area where the targets are generated. The first one, denoted as LD (Large Distance), consists of an area of $200 \times 200$ miles, while the second and the third ones, denoted as ND (Normal Distance) and SD (Short Distance), measure $80 \times 40$ miles and $40 \times 40$ miles, respectively. The results are reported in Table 1 where Cases \# denotes the number of samples considered and Opt. Sol. the percentage of cases in which the sequences generated by exactly solving the CV-TSP problem and by using the E-TSP heuristic coincide. Finally, Avg Degradation and Max Degradation denote respectively the average and the maximal degradations in the cost of E-TSP w.r.t. the optimal cost of CV-TSP. Note that the performance of the proposed heuristic is, at least in this case, a very tight approximation of the optimal solution. It is possible also to note that, as expected, for points generated in a larger area the heuristic and the optimal CV-TSP solutions coincide in almost all cases while for points very close each others the average cost degradation increases. In Table 2, the statistics of the number of samples showing degradation levels respectively lower than $0.1 \%, 1 \%$, $2.5 \%, 5 \%$ and $10 \%$ are reported. As a final remark we want to point out the fact that cases in which the cost degradation is greater than $1 \%$ usually correspond to situations where several points are very close one to each other: for instance the outlier in the SD case with a degradation of $25.1 \%$ corresponds to the case in which 4 out of the 5 points to be visited, as depicted in Figure 3 , are within a ball of radius 8.1 miles from the starting point.

\begin{tabular}{|c|c|c|c|c|c|}
\hline Degradation & $<0.1 \%$ & $<1 \%$ & $<2.5 \%$ & $<5 \%$ & $<10 \%$ \\
\hline LD & $94.2 \%$ & $99 \%$ & $100 \%$ & $100 \%$ & $100 \%$ \\
ND & $92.0 \%$ & $97.9 \%$ & $99 \%$ & $99.5 \%$ & $100 \%$ \\
SD & $82.4 \%$ & $88.6 \%$ & $93.2 \%$ & $97 \%$ & $99.4 \%$ \\
\hline
\end{tabular}

Table 2 Number of cases with degradation level lower than $0.1 \%, 1 \%, 2.5 \%, 5 \%$ and $10 \%$ 

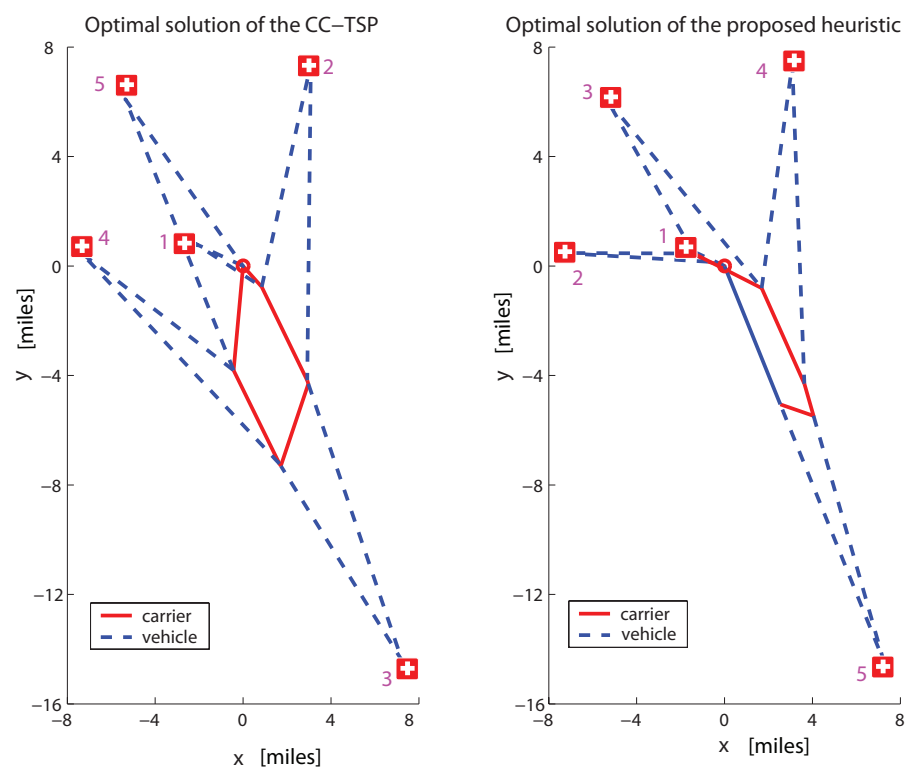

Fig. 3 Comparisons between the optimal CV-TSP solution and the solution obtained by means of the proposed heuristic. Numbers represent the visiting order.

\section{Conclusions}

This note has addressed the Traveling Salesman Problem for a class of carrier-vehicle systems in which a slow carrier with infinite operating range cooperates with a faster vehicle which, on the contrary, has a limited operating range. A heuristic solution has been proposed and an analytical bound on its conservativeness has been derived. Extensive numerical simulations have given an insight of the effectiveness of the proposed method in some cases of interest.

\section{Acknowledgments}

This paper was supported by the Belgian Network DYSCO (Dynamical Systems, Control and Optimization) funded by the Interuniversity Attraction Poles Program, initiated by the Belgian State, Science Policy Office. This paper was also supported by the Young Researcher Grant for the research project "Path Planning for Heterogeneous Cooperating Vehicles", D.R. n. 1054 del 27.4.2009, University of Calabria, Italy and by the collaborative project AIRobots (Innovative Aerial Service Robots for Remote inspections by contact, ICT 248669) supported by the European Community under the 7th Framework Programme. The scientific responsibility rests with its authors. The authors want to thank prof. E. Frazzoli of Massachusetts Institute of Technology the many 
useful discussions and suggestions. A special thanks to Dr. K. Garnett of BC for the inspiration.

\section{References}

[1] R.M. Murray, "Recent Research in Cooperative Control of Multi-Vehicle Systems," in ASME Journal of Dynamic Systems, Measurement, and Control, vol. 129, no. 5, pp. 571-583, 2007.

[2] P. R. Chandler, M. Pachter, D. Swaroop, J. M. Fowler, J. K. Howlett, S. Rasmussen, C. Schumacher, and K. Nygard, "Complexity in UAV cooperative control," in American Control Conference, (Anchorage, AK), pp. 1831-1836, 2002.

[3] R. W. Beard, T. W. McLain, M. A. Goodrich, and E. P. Anderson, "Coordinated target assignment and intercept for unmanned air vehicles," IEEE Transactions on Robotics and Automation, vol. 18, no. 6, pp. 911-922, 2002.

[4] S. Darbha, "Combinatorial motion planning for a collection of Reeds-Shepp vehicles," tech. rep., ASEE/AFOSR SFFP, AFRL, Eglin, 2005.

[5] A. E. Gil, K. M. Passino, and A. Sparks, "Cooperative scheduling of tasks for networked uninhabted autonomous vehicles," in IEEE Conf. on Decision and Control, (Maui, Hawaii), pp. 522-527, Dec. 2003.

[6] L. E. Parker, "Multiple Mobile Robot Systems,"Springer Handbook of Robotics," Springer, Ed. By B. Siciliano and O. Khatib, pp. 921-936, 2008.

[7] R. R. Murphy, S. Tadokoro, D. Nardi, A. Jacoff, P. Fiorini, H. Choset and A. M. Erkmen.. "Search and rescue robots," Springer Handbook of Robotics. Springer, Ed. By B. Siciliano and O. Khatib, pp. 1151-1171, 2008.

[8] YU Cao, AS Fukunaga, A Kahng. "Cooperative mobile robotics: Antecedents and directions," Autonomous robots, pp. 226-234, Springer, 1997.

[9] S. M. LaValle, Planning Algorithms. Cambridge, UK: Cambridge University Press, 2006.

[10] E. Frazzoli and F. Bullo, "Decentralized algorithms for vehicle routing in a stochastic time-varying environment," in IEEE Conf. on Decision and Control, (Paradise Island, Bahamas), pp. 3357-3363, Dec. 2004.

[11] D. J. Bertsimas and G. J. van Ryzin, "A stochastic and dynamic vehicle routing problem in the Euclidean plane," Operations Research, vol. 39, pp. 601-615, 1991.

[12] Garone E., Naldi R., Casavola A., Frazzoli E. "Cooperative path planning for a class of carrier-vehicle systems", 47th IEEE Conference on Decision and Control, December 10-11, Cancun, Mexico, 2008.

[13] S. Arora, "Polynomial-time Approximation Schemes for Euclidean TSP and other Geometric Problems", Journal of the ACM 45(5), pp. 753-782, 1998. 